\title{
Wege aus dem Misstrauen
}

\author{
Theoretische und praktische Überlegungen ${ }^{1}$
}

Wenn man im Kontext der Frage nach dem Verhältnis einer religiösen Minderheit zu einer religiösen Mehrheit die Vertrauensthematik ansprechen soll, liegt die Vermutung nahe, dass das Verhältnis der Minderheit zur Mehrheit häufig von Misstrauen getragen ist. Bezieht man die Fragestellung auf den deutschen und den türkischen Kontext, scheint etwa die Annahme im Hintergrund zu stehen, dass das Verhältnis vieler Muslime zur deutschen Mehrheitsgesellschaft ebenso als misstrauisch gekennzeichnet werden kann wie das Verhältnis vieler Aleviten zur türkischen Mehrheitsgesellschaft. Ich will hier nicht beurteilen, inwieweit die Annahme eines solchen Misstrauens in beiden Fällen gerechtfertigt ist, denn das ist eher eine empirische Frage, über die ich wenig Auskunft geben kann. Auch will ich nicht beurteilen, wie sinnvoll es ist, die Lage der Muslime in Deutschland mit der Lage der Aleviten in der Türkei zu vergleichen. Mir kommt es nur darauf an, den Fokus zunächst in Abstraktion von den konkreten Konflikten stärker auf das Vertrauen und das Misstrauen zu lenken, um die Frage zu klären, was genau eigentlich gemeint ist und was auf dem Spiel steht, wenn wir von Misstrauen und von Vertrauen reden. Auf den ersten Blick könnte es so scheinen, als sei sehr klar, was hier auf dem Spiel steht: Misstrauen ist schlecht, Vertrauen ist gut, also müssen wir darüber nachdenken, wie wir das Misstrauen überwinden können, um mehr vertrauensvolles Miteinander zu erreichen. Und natürlich würde ich nicht bestreiten, dass man den Sachverhalt so schildern kann. Dennoch lässt sich, so meine ich, einiges lernen, wenn man etwas näher hinschaut, und

\footnotetext{
1 Vortrag gehalten auf dem VIII. Tarabya-Forum in Istanbul im Juni 2010. Seit 2002 veranstaltet die deutsche Botschaft in Ankara im (Haus des deutsch-türkischen Dialogs in Tarabya (Istanbul) die Konferenzreihe sIslam und Europa als Thema der deutsch-türkischen Zusammenarbeit). Ziel der Tarabya-Konferenz ist es, zur interkulturell besseren Verständigung zwischen Deutschland und der Türkei beizutragen. Ein besonderer Fokus liegt auf religiösen Fragen. Thema des VIII. Forums waren «Neue Formen des Dialogs zwischen Staat und Zivilgesellschaft in Deutschland und in der Türkei: die Deutsche Islamkonferenz und der Aleviten Çalistay). Die Aleviten bilden neben den sunnitischen Muslimen die größte religiöse Gruppierung in der Türkei und kämpfen um eine Verbesserung ihres religiösen Status.
} 
genau das will ich hier versuchen. In einem ersten Schritt (I.) will ich dabei einige begriffliche Überlegungen anstellen, die helfen sollen, den Phänomenbereich des Vertrauens und Misstrauens besser zu überschauen. Anschließend (II.) möchte ich versuchen, diese eher abstrakten Überlegungen mehr mit Leben zu füllen, um Ihnen an einzelnen Beispielen zu veranschaulichen, was nötig ist, um eine Situation des Misstrauens zu überwinden. Das klingt sehr viel versprechend, und ich sollte hier vorsichtiger formulieren, denn ich bin kein policy-maker, der unter Entscheidungsdruck steht, sondern ein politischer Philosoph, der sich den Luxus einer größeren Distanz zum Alltagsgeschäft der Politik erlauben kann. Ich will Ihnen nur einige Vorschläge skizzieren, die im Rahmen der politischen Theorie selbst gemacht worden sind, um mit Situationen großen Misstrauens unter den Bürgern eines Gemeinwesens (nennen wir dies bürgerschaftliches Misstrauen) oder zwischen einzelnen Bürgern und den staatlichen Instanzen dieses Gemeinwesens umzugehen (nennen wir dies politisches Misstrauen).

I.

Ich hatte behauptet, Vertrauen sei besser als Misstrauen, aber das ist natürlich nicht richtig. Es kann Situationen geben, in denen es ganz verständlich und sogar rational ist, eher misstrauisch zu sein als vertrauensvoll. Vertrauen ist also nur unter bestimmten Umständen angebracht, unter anderen sollte man eher misstrauisch sein. Sowohl für Vertrauen als auch für Misstrauen aber gilt, dass wir es mit Einstellungen zu tun haben, die man als engagiert betrachten kann. Wir wollen etwas, wir haben ein Ziel, und es gibt verschiedene Wege, auf denen man das Ziel erreichen kann. Wir können ein Ziel auf vertrauensvolle Weise erreichen, wir können es aber auch erreichen, wenn wir misstrauischer sind. Manche Ziele freilich kann ich nicht erreichen, wenn mein Misstrauen mich daran hindert, dem Ziel überhaupt nachzugehen; aber auch hier gilt, dass mein Misstrauen anzeigt, dass ich das Ziel gerne erreichen möchte. Das meine ich, wenn ich von engagierten Einstellungen spreche: Unabhängig von allen Unterschieden sindVertrauen und Misstrauen darin verbunden, dass sie Einstellungen sind, in denen sich ein Interesse am Erreichen von Zielen offenbart, die uns wichtig sind. In dieser Hinsicht stehen Vertrauen und Misstrauen also nicht im Gegensatz zueinander, ihr gemeinsamer Gegensatz wäre eher so etwas wie eine Einstellung der Gleichgültigkeit oder Apathie. Misstrauen ist im Lichte dieser 
Überlegungen vielleicht immer noch ein Problem, aber es ist immerhin klar, dass der Misstrauische unter Umständen ähnliche Ziele wie derVertrauensvolle verfolgt, nur weiß er nicht, wie er sie erreichen kann oder ob er sie überhaupt erreichen kann. Ich erwähne diesen Punkt, weil man Misstrauen häufig als kontradiktorischen Gegensatz von Vertrauen konstruiert. Das ist nicht der Fall. Wer ohne Vertrauen ist, ist nicht unbedingt misstrauisch, er kann auch einfach einem Thema oder Problem ganz gleichgültig begegnen. Misstrauen ist gewissermaßen der erste Schritt auf ein Ziel zu und kann deswegen durchaus auch positiv betrachtet werden.

Wenn ich annehme, dass Vertrauen und Misstrauen engagierte Einstellungen sind, in denen sich ein Interesse an bestimmten Zielen offenbart, dann impliziere ich damit, dass beide Einstellungen nicht hinreichend beschrieben sind, wenn man sie nicht in Verbindung mit diesen Zielen diskutiert. So wertvoll es etwa sein mag, einem anderen zu vertrauen, wir können immer auch fragen, was für ein Handeln durch dieses Vertrauen möglich wird (und durch Misstrauen erschwert oder gar unmöglich wird). Vertrauen hat einen Eigenwert, denn wenn wir vertrauen, erkennen wir jemanden an als einen, dem vertraut werden kann, und das ist ein Status, der für viele durchaus erstrebenswert ist und der nicht einfach durch andere Einstellungen substituiert werden kann. Aber neben diesem Eigenwert lässt sich auch fragen, was für ein Handeln uns Vertrauen ermöglicht. Wenn ich jemandem mein Kind anvertraue, kann ich ins Kino gehen, sonst könnte ich es nicht oder müsste mir andere Wege überlegen. Die Einstellung des Vertrauens hilft mir also, Ziele $\mathrm{zu}$ erreichen, die wertvoll für mich sind und deren Wert durchaus unabhängig vom Wert des Vertrauens bestimmt werden kann. Warum mag ich es, ins Kino zu gehen? Weil ich Erholung brauche, weil ich meine Vorstellungskraft bilden möchte, weil ich das als Teil meiner Selbstverwirklichung betrachte etc. - wie immer man hier genauer argumentiert, ich kann dieses Ziel erreichen, weil ich vertraue, aber der Wert dieses Ziels hängt nicht unbedingt am Wert des Vertrauens. Allerdings mag es Ziele geben, die gleichsam näher mit demVertrauen zusammen hängen oder die nur imVertrauen erreicht werden können. Ohne Vertrauen kann man sich beispielsweise kaum Freundschaft vorstellen und auch Liebe zwischen Menschen scheint wesentlich mitVertrauen zu tun zu haben. Das heißt aber nicht, dass es in diesen Beziehungsformen nicht doch auch um anderes als um Vertrauen geht. Auch wenn Vertrauen wesentlich oder sogar konstitutiv für solche Beziehungen ist, versuchen wir Freundschafts- und 
Liebesbeziehungen in der Regel aus vielfältigen Gründen aufzubauen, und Vertrauen ist hier keinesfalls der einzige Grund.

Ich habe Vertrauen bislang als eine engagierte Einstellungen beschrieben, die uns in die Lage versetzt, Zielen nachzugehen, die uns wichtig sind. Ich habe noch nicht gesagt, wie sich Vertrauen aufbaut und wem wir es schenken. Es ist klar, dass sich in diesen einfachen Fragen viele komplexe Aspekte verbergen, die ausführlicher behandelt werden müssten. Ich will meine Überlegungen aber hier schon auf die an diesem Punkt relevante Themenstellung zuschneiden. Grob gesprochen schenken wir dem Vertrauen, von dem wir erwarten, dass er uns beim Erreichen unserer Ziele in kooperativer Weise unterstützt. Das heißt nicht unbedingt, dass er diese Ziele teilen muss, aber es heißt, dass die Tatsache meines Vertrauens, wenn sie denn irgendwie erkennbar ist, ein kooperatives Verhalten nach sich zieht. Mein Vertrauen lässt ihn nicht kalt, er berücksichtigt es in seiner Reaktion auf mich. Der Vertrauende wiederum setzt zwar auf dieses kooperative Verhalten, aber es bleibt immer ein Restrisiko, da die Reaktion des anderen nicht programmiert werden kann und im Vertrauen auch gar nicht programmiert werden soll. Wer absolute Sicherheit will, braucht kein Vertrauen. Jemandem im Vertrauen als einen anzuerkennen, dem vertraut werden kann, heißt, ihm einen Ermessensspielraum einzuräumen, der es ihm erlaubt, mein Vertrauen so oder anders zu berücksichtigen. Es geht hier nicht um totale Kontrolle, denn das würde das Verhältnis ganz und gar ändern und würde auch die normativen Gewinne tilgen, der mit Vertrauensverhältnissen in der Regel verbunden sind.

Wichtig ist die Formulierung, dass die Kooperationserwartung besteht, auch wenn der, der mit uns kooperieren soll, die eigenen Ziele nicht teilt. Gerade im Kontext von Religion entsteht genau hier ein großes Problem, da Religionen oft mit absoluten Wahrheitsansprüchen einhergehen, die wenig Raum zu lassen scheinen für Wahrheitsansprüche, die mit anderen Religionen einhergehen. Der erste Schritt zu gegenseitigem Vertrauen zwischen Religionen besteht dann darin, überhaupt erst einmal die Existenzberechtigung der je anderen Religion einzuräumen, ohne davon auszugehen, dass die Wahrheitsansprüche der anderen Religion die eigenen Wahrheitsansprüche notwendig entwerten oder gefährden. Ich kann nicht ausführlich auf diese Thematik eingehen, aber was sich hier zeigt, ist noch einmal, wie voraussetzungsreich stabile Verhältnisse gegenseitigen Vertrauens sind. Wer dogmatisch an den Wahrheitsannahmen der eigenen Religion festhält, wird vermutlich nur dann vertrauensvoll mit anderen Religionen umgehen wollen, 
wenn sich im eigenen Religionsverständnis genügend begriffliche Ressourcen finden, die einen respektvollen Umgang mit anderen Religionen erlauben. Selbst Toleranz mag als Haltung nicht sonderlich zuträglich für vertrauensvolle Einstellungen sein, weil sie durchaus mit ablehnenden Bewertungen der je anderen Religion verbunden ist (ich lehne diese Religion ab, trotzdem dulde ich sie, weil...). Es ist ein Unterschied, ob ich die Ziele des anderen einfach nur nicht teile oder ob ich sie ablehne - und dieser Unterschied dürfte für die Frage nach dem gegenseitigen Vertrauen nicht ganz unerheblich sein.

Ich habe am Anfang so getan, als ginge es mir zunächst um Vertrauensverhältnisse zwischen zwei Personen, bin dann aber langsam zur Frage der Religion übergegangen. Wenn wir vom Verhältnis einer minoritären Religion zu einer Mehrheitsreligion sprechen oder wenn wir uns mit dem Vertrauen der Bürger zum Staat beschäftigen, haben wir es ja nicht mit einem Vertrauen unter Einzelnen zu tun, sondern mit einem Vertrauen kollektiver Akteure untereinander oder zu einer anderen kollektiven Instanz. Natürlich agieren nicht Religionen oder «der〉 Staat, sondern die Religionsmitglieder und die den Staat repräsentierenden Personen, aber ihr Handeln wird häufig so betrachtet, als symbolisiere es die Einstellungen des Kollektivs, in dessen Namen sie jeweils agieren. Für die Vertrauensthematik ergibt sich hier ein (weiteres) enormes Problem: Hinter jedem einzelnen Akteur steht symbolisch ein Kollektiv, das gleichsam in Haft genommen wird für die Handlungen dieses Akteurs, auch wenn faktisch nur dieser Akteur gehandelt hat. Nur weil dies so ist, kann es sein, dass das Handeln einzelner Akteure das Vertrauen in «den〉 Staat oder 〈die〉 islamische oder 〈die» christliche Religion untergräbt. Für die kollektiven Akteure ergibt sich daraus die Schwierigkeit, dass sie sich einerseits zwar offiziell darum bemühen können, gegenüber anderen Akteuren vertrauenswürdig und verlässlich zu sein, dass sie andererseits aber für das Fehlverhalten Einzelner in Haft genommen werden, obwohl sie konkret gar nichts mit diesem Verhalten zu tun haben. Gleichzeitig gilt natürlich, dass es häufig ganz unangemessen ist, so zu tun, als gäbe es jeweils ein Kollektiv. Die Aleviten, das habe ich gelernt, sind durchaus gespalten, was die Frage angeht, ob sie dem Islam zugehören. Andererseits fühlt sich auch nicht jeder offiziell dem Islam zugerechnete Deutsche als Muslim oder teilt die Auffassung der offiziellen Islamverbände. Aber auch das schützt nicht vor verschiedenen Formen der Kollektivhaftung. Ich werde auf dieses Problem zurückkommen. 
II.

Ich will nun zum zweiten Teil meines Vortrags kommen. Ich hatte angekündigt, darüber zu sprechen, wie ein Zustand des Misstrauens überwunden werden kann. Nach meinen bisherigen Ausführungen sollte nun deutlich sein, dass zunächst einmal geklärt werden sollte, ob es sinnvoll ist, die Haltung des Misstrauens zu überwinden. Sie mag mit vielen Problemen einhergehen, aber sie kann auch rational sein, kann die angemessene Reaktion auf eine als misslich empfundene Lage sein, die tatsächlich misslich ist. Bevor also direkt gefragt wird, wie sich das Misstrauen überwinden lässt, sollte gefragt werden, warum der Misstrauische misstrauisch ist, welche Ziele der Misstrauische erreichen will (aber nicht erreichen kann oder nicht so erreichen kann, wie er es gerne erreichen möchte) und was es bedeuten würde, diese Ziele in vertrauensvoller Einstellung zu erreichen. Es mag merkwürdig klingen, aber konkret heißt das, dass das Misstrauen einer Gruppe erst einmal ernst genommen werden sollte und auf seine Berechtigung hin befragt werden muss. Dies lässt sich auch deswegen nicht umgehen, weil Misstrauen häufig auf Erfahrungen der Verletzung eigener Ansprüche und Erwartungen basiert, deren Spuren in psychologisch tiefe Schichten der Persönlichkeit reichen können. Man kennt dieses Problem aus der Diskussion über Wahrheitskommissionen in Südafrika: Bevor überhaupt Schritte zum Abbau des Misstrauens erfolgen können, gilt es die negativen Erfahrungen, die das Misstrauen in sich bündelt, zur Sprache zu bringen. Es kann ein erster Schritt weg vom Misstrauen sein, die Quellen des Misstrauens offen anzuerkennen, denn die Weigerung, Ungerechtigkeit oder Diskriminierung zu erinnern und damit als Ungerechtigkeit und Diskriminierung anzuerkennen, läuft Gefahr, die Gründe des Misstrauens am Leben zu lassen; wird doch diese Weigerung nur wie ein weiterer Akt der Benachteiligung oder Diskriminierung erfahren, der im schlimmsten Fall Ressentiments oder sogar Rachegefühle schürt.

Man kann sich leicht klar machen, dass dieser Schritt der Anerkennung der Gründe des Misstrauens ein erheblicher Schritt ist. Michael Ignatieff beschreibt das Gewicht der Vergangenheit im Zusammenhang mit den Konflikten im ehemaligen Jugoslawien einmal so: «[T] he past continues to torment because it is not the past. [...] Reporters in the Balkan war often observed that when they were told atrocity stories they were occasionally uncertain whether these stories had occured yesterday or in 1941, 1841, or 1441.» Die vergangenen Verbrechen sind gleichsam eingeschlossen, so schreibt er 
weiter, in einer «ewigen Gegenwart». ${ }^{2}$ Ein Offenlegen der alten und doch gegenwärtigen Wunden wird auch deswegen schmerzhaft sein, weil dieser Prozess natürlich auch deutlich machen könnte, warum die eine Seite der anderen mit Hass, Ablehnung oder Verachtung begegnet, aber auch dies wird Teil des Versuchs sein, Misstrauen zu überwinden.

Ich habe damit natürlich nur einen ersten Schritt benannt. Was aber soll das Ziel der Überwindung von Misstrauen sein? Und was soll das Ziel des Vertrauens sein, wenn es denn hergestellt ist? Wer soll wem vertrauen? Diese Fragen mögen merkwürdig klingen, weil am Wert von Vertrauen kaum gezweifelt werden kann, aber ich stelle sie trotzdem, weil es wenig hilfreich ist, abstrakt zu postulieren, dass Vertrauen gut sei, unabhängig von den praktischen Kontexten, in denen sich dann das Vertrauen bewähren soll. In ihrem 2003 erstellten Gutachten $^{3}$ zur Frage, ob die in Deutschland lebenden Aleviten als Religionsgemeinschaft im Sinne des Art. 7,Abs. 3 des Grundgesetzes gelten können, schreibt die Religionswissenschaftlerin Ursula Spuler-Stegemann:

«Vom türkischen Staat fordern die Aleviten die Anerkennung als gleichberechtigte Staatsbürger. Sie wollen eigenen Religionsunterricht oder wenigstens angemessene Berücksichtigung der alevitischen Lehre im staatlichen Religionsunterricht, eigene Sendezeiten in Rundfunk und Fernsehen, Beendigung der Politik des Zu-Sunniten-Machens»,

und, so Spuler-Stegemann weiter, "Gleichbehandlung mit den Sunniten bei derVergabe staatlicher Gelder». Das sind sehr konkrete Forderungen und Ziele, und man könnte fragen, inwieweit zu ihrer VerwirklichungVertrauen notwendig ist. Eher wird es nötig sein, zur Erreichung dieser Ziele zivilgesellschaftliche Ressourcen zu mobilisieren, die in der Lage sind, kontinuierlich und enttäuschungsresistent Druck auf die offiziellen politischen Organe auszuüben, die für die Umsetzung der genannten Ziele Verantwortung tragen. Vertrauen wird hier erst ins Spiel kommen, wenn einige der erstrebten Ziele erreicht sind, wenn es etwa eine konstitutionelle Anerkennung einer minoritären Religion gibt oder wenn es gelungen ist, eine offizielle Repräsentation dieser Religion zu etablieren, die stellvertretend die

2 Zitiert nach M. Minow, Breaking the Cycles of Hatred. Memory, Law, and Repair, Princeton 2002, 28

3 Verfasst für das Ministerium für Schule, Jugend und Kinder in Nordrhein-Westfalen, vgl. http://www.alevi-nrw.com/index.php?option=com_docman\&task=doc_ download $\&$ gid $=15 \&$ Itemid $=10$. 
Interessen ihrer Mitglieder wahrnimmt. Politische Kämpfe beruhen auf der Annahme, dass kein anderer die eigenen Interessen vertreten wird und stehen insofern in der Mitte zwischen Misstrauen und Vertrauen. Sie sind Ausdruck eines Zweifels an den bestehenden Institutionen und bemühen sich zugleich darum, einen Zustand herzustellen, in dem dann Vertrauen als Einstellung überhaupt erst relevant werden kann.

Wenn diese Überlegungen stimmig sind, dann stellt sich die Frage desVertrauens in politischen Kontexten häufig erst, wenn für die, die um ihre Rechte und Interessen kämpfen, eine Menge erreicht ist. Nehmen wir etwa an, einer religiösen Minderheit werden bestimmte für einen politischen Raum wichtige Rechte oder Privilegien gewährt. Nehmen wir darüber hinaus an, sie hat es geschafft, politische Repräsentation in den relevanten nationalen und transnationalen Institutionen zu erreichen. Dies wird nötig sein, da im Kontext einer religiös und kulturell gespaltenen Gesellschaft davon auszugehen ist, dass politische Vertreter der religiösen oder kulturellen Mehrheit nicht unbedingt in der Lage sein werden, die spezifischen Bedürfnisse und Interessen einer Minderheit angemessen zu berücksichtigen. Tatsächlich weist das Problem über den engeren Kontext parlamentarischer Repräsentation hinaus. Nehmen wir nur die Anzahl von Mitarbeitern mit migrantischem Hintergrund in gehobenen Positionen der Bundesrepublik. Özlem Topcu schreibt in der Zeit:

«Nicht einmal ein Prozent der fest angestellten Zeitungsredakteure haben $[\ldots]$ einen Migrationshintergrund. Im öffentlichen Dienst sieht es nicht viel anders aus, dort beträgt der Anteil der Mitarbeiter mit Zuwanderungsgeschichte etwa drei Prozent.» ${ }^{4}$

Aber selbst, wenn Fortschritte erzielt sind, könnte man nun sagen, dass immer noch nicht der Zeitpunkt für Vertrauen gekommen ist, weil man besser in kontrollierender Absicht darauf achtet, ob die verbrieften Rechte auch tatsächlich praktisch wirksam umgesetzt werden oder ob die Repräsentanten die eigenen Interessen tatsächlich in den politischen Beratungsprozessen berücksichtigen. Gibt es nicht in Demokratien eingebaute Mechanismen des Misstrauens, die einen Missbrauch von Ämtern und Positionen verhindern sollen? Abgeordnete, die unsere Interessen nicht angemessen vertreten, können wir abwählen. Auf Gerichtsurteile, die das Recht einseitig oder diskriminierend auslegen, können wir mit öffentlichem Druck oder öffentlicher Empörung reagieren. Aber genau hier kommen

${ }_{4}$ Ö.Topcu, Weiß, männlich, Elite, in: Die Zeit 22, 27. Mai 2010, 12. 
die Grenzen verfassungsförmiger oder institutioneller Versuche des Verzichts auf Vertrauen zum Vorschein. $\mathrm{Ob}$ nämlich ein solcher Protest Erfolg hat, wird nicht zuletzt daran hängen, wie viele Mitbürger ihn teilen, auch wenn ihre eigenen Interessen nicht unmittelbar berührt zu sein scheinen. Wie erfolgreich eine Verfassung ist, wie erfolgreich rechtliche Regelungen umgesetzt werden, hängt also an der Existenz eines egalitären bürgerschaftlichen Status. Und ein solcher Status kann nur bedingt rechtlich hergestellt werden, denn was immer rechtlich oder verfassungsförmig etabliert ist, kann nur praktisch wirksam werden, wenn es innerhalb der Bürgerschaft die Bereitschaft gibt, über die eigenen Interessen hinweg für die Rechte anderer einzutreten. Man kann dann sagen, das politische Vertrauen setzt ein bürgerschaftliches Vertrauen voraus, dessen Voraussetzung wiederum die Wahrnehmung von Gemeinsamkeiten ist, die gegebene Differenzen auch in der Wahrnehmung der Betroffenen transzendieren. ${ }^{5}$

Damit bin ich also beim Vertrauen angelangt. Ich habe bis jetzt gleichsam versucht anzudeuten, wann Vertrauen in politischer Hinsicht tatsächlich ins Spiel kommt, und ich habe in Anlehnung an meine anfänglichen Überlegungen ein politisches und ein bürgerschaftliches Vertrauen unterschieden. Locke hat bekanntlich das politische Vertrauen in einflussreicher Weise als eine Art Treuhandverhältnis bestimmt. Die Legislative sei nur eine Gewalt, so Locke, «die auf Vertrauen beruht und zu bestimmten Zwecken handelt». ${ }^{6}$ Erfüllt sie ihren Zweck nicht, dann kann das Vertrauen entzogen werden. Für Locke besteht dieser Zweck der Regierung wesentlich in der Freiheits- und Eigentumssicherung, wir dagegen würden heute vielleicht etwas allgemeiner davon sprechen, dass wir von der Regierung, aber auch von den parlamentarischen Repräsentanten die Wahrung unserer Interessen erwarten, und zwar der Interessen, von denen wir mit guten Gründen sagen können, dass sie politisch relevant sein sollten. Damit sich zwischen den Bürgern und ihren Repräsentanten so etwas wie ein treuhänderisches Vertrauensverhältnis einstellen kann, ist vorgeschlagen worden, dass vor allem die gesellschaftlichen Gruppen, die auf eine lange Geschichte der Diskriminierung verweisen, eigene Kandidaten in die Parlamente schicken dürfen, und das scheint mir auch ein legitimer Vorschlag zu sein, eine gewisse Responsivität zwischen den Abgeordneten und ihren Wählern herzustellen. Andererseits liegen hier natürlich mehrere Probleme

5 Siehe M.S. Williams, Voice, Trust, and Memory. Marginalized Groups and the Failings of Liberal Representation, Princeton 1998, 168ff.

6 J. Locke, Zwei Abhandlungen über die Regierung, Frankfurt a.M. 1977, 293 f. 
verborgen, denn viele Gruppen sind in sich gespalten und lassen sich keinesfalls über einen Interessenkamm scheren. Es gibt nicht, darauf spielte ich schon an, die Aleviten oder die deutschen Muslime. Ich kann nicht auf diese komplexe Problematik eingehen, aber sie verändert auch noch einmal ihr Gesicht, wenn das bürgerschaftliche Vertrauen thematisch wird, also das Vertrauen der Bürger untereinander. Dieses Vertrauen basiert im Grund schlicht darauf, dass ich als einzelner das Gefühl der Zugehörigkeit zur politischen Gemeinschaft habe, zu der ich zunächst qua Aufenthaltsort oder auch durch Zuerkennung bestimmter grundlegender Rechte gezählt werde. Fehlt dieses Zugehörigkeitsgefühl, ist in der Regel Misstrauen das Ergebnis. Ich will das an einem Beispiel veranschaulichen, das sich mit dem Verhalten türkischer Migranten zur deutschen Polizei beschäftigt. Der Soziologe Norbert Schröer hat Gespräche verglichen, die deutsche Polizisten mit türkischen Migranten und mit deutschen Beschuldigten geführt haben. Überraschend kam dabei heraus, dass türkischstämmige Beschuldigte oft weniger streng bestraft wurden als deutsche, weil sie im Verlauf des Verfahrens viel weniger kooperieren und häufig schlicht die Aussage verweigern. Diese Verweigerungshaltung hat ihre Quelle allerdings in einem ausgeprägten Misstrauen der türkischstämmigen Beschuldigten gegenüber der deutschen Polizei. Sie gehen nämlich von Anfang an davon aus, dass sie kein faires Verfahren erhalten, was wiederum daran liegt, dass sie nicht glauben, ein «selbstverständliches Mitglied der 〈Residenzgesellschaft»» zu sein. Jedem einzelnen beschuldigten Migranten stellt sich die Frage, so Schröer,

$$
\begin{aligned}
& \text { "ob er im gesellschaftlichen Leben als gleichberechtigter Kom- } \\
& \text { munikationspartner akzeptiert wird, ob er die Gelegenheit } \\
& \text { bekommt, seine Perspektive, seine ihm eigene Lebensform zur } \\
& \text { Geltung zu bringen und mit ihr Anschluss an die aufnehmen- } \\
& \text { de Gesellschaft zu finden. In der Beantwortung dieser Fragen } \\
& \text { entscheidet sich, ob der Migrant Loyalitäten und Vertrauen zur } \\
& \text { gastgebenden Gesellschaft aufbauen [...] kann». }
\end{aligned}
$$

Vertrauen, so hieß es, gilt jenen, von denen wir erwarten, dass sie unsere Ziele in kooperativer Weise unterstützen, auch wenn diese anderen diese Ziele nicht teilen. Das Empfinden, kein Bürger (und hier kann man dann auch einfügen: keine Religion) zweiter Klasse zu sein, kann ein Vertrauen in diesem Sinne erzeugen. Dieses Empfinden jedoch ist nicht durch Rechtsbeschlüsse herzustellen. Eher muss es im

\footnotetext{
7 N. Schröer, Verfehlte Verständigung? Kommunikationssoziologische Fallstudie zur interkulturellen Kommunikation, Konstanz 2002, 243.
} 
alltäglichen Kampf um die Anerkennung der eigenen Lebensform erzeugt werden. Sowohl die Bereitschaft zu vertrauen, als auch die Bereitschaft, sich als vertrauenswürdig zu erweisen, wird in dem Maße steigen, in dem ich die Erfahrung gemacht habe, dass meine Fähigkeiten und Ziele, aber auch meine Rechte, nicht nur geduldet werden, weil ihre Verletzung mit Sanktionen verbunden wäre. Vielmehr muss eine Art sozialer Anerkennung wirksam werden, die sich darin äußert, dass andere meine Ziele auch dann unterstützen, wenn sie sie nicht teilen. Der Weg zu dieser Form des Vertrauens zu den Mitbürgern ist lang, und es gibt auch keine Patentantworten, die schnell darüber Auskunft geben, wie man ihn genau zu beschreiten hat. Auch ist vielleicht noch immer nicht wirklich geklärt worden, welche Ziele denn mit dem Vertrauen verbunden sind. Aber die Klärung dieses Punkts lässt sich ebenfalls nicht am Schreibtisch erzielen, sie ist Gegenstand der demokratischen Auseinandersetzung, an der auch wir zur Zeit beteiligt sind. 